\title{
The Technique and Craftsmanship of Tattooing in the Professional Tattoo Parlour
}

Lars Kristensen

DTL - Danish Tattooist Assoc., Sorø, Denmark standing of good hygiene, personal as well as for the shop room in which the tattoos are made, have been made.

\section{Initial Customer Contact}

The work process of a modern professional tattoo artist today starts at first contact with the customer. This could be over the phone, internet or in person.

From the beginning, it's important to make it clear to the customer that you, as a professional tattoo artist, take the customer's wishes and concerns seriously.

The professional tattoo artist takes time to guide and inform the customer about the known risks of using colour in a tattoo. Likewise, he or she informs the customer about aftercare of the tattoo: what you should do and what you shouldn't, and which products should be used, over the short- and long-term, when you take care of your tattoo. \begin{abstract}
shop room, as well as improvements in the underunder the skin of the customer, the same today as it was in 1891.

Since then, many technical improvements to other equipment that the tattoo artist uses, such as needles, colours, aftercare products, and the

The kind of tattooing described in this chapter is

This kind of tattooing has, in many ways, been unchanged since its patenting on the 8th of December 1891 . The tattoo is coloured by getting the art.

Abstract

al tattoo artist needs to have talent for composing and drawing motives - the artistic part of his or her profes-

\section{Condition}




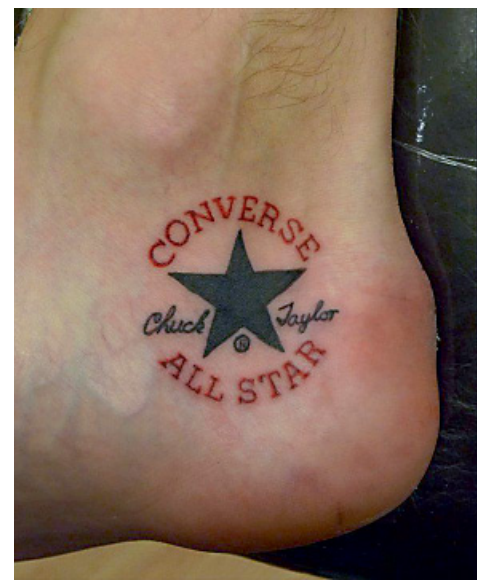

Fig. 1. Craftsmanship is not only about big tattoos. Sometimes, it's the detail that counts.

It's also very important to advise the customer regarding design, location, size, etc. (fig. 1). It's also a part of the work to say 'no' to tattoos that can't be made professional and satisfying, for example, tattoos on fingers.

A serious tattoo artist should also express his or her own subjective evaluation of a tattoo project. If the project is so controversial that the customer would get reactions that he or she did not expect from others, the tattoo artist should speak up. Then, the customer, based on the feedback from the tattoo artist, could decide whether he or she still wants the tattoo.

\section{In the Shop}

When a customer shows up in a shop to get a tattoo, the person - if he has chosen a professional tattoo artist, will enter a shop that is adapted to its purpose - the making of tattoos.

You will see many examples of shops that sell, for example, clothing or equipment. If this is the case, it's very important that the areas are clearly separated, so there is no doubt about where the tattoos are made. Also, this section should be ar- ranged so the cleaning can be done in a proper and responsible manner.

You'll see that many tattoo artists have a tendency to collect souvenirs from their numerous trips around the world to tattoo conventions. Here, the same policy applies: the area where the tattoos are made should be easy to clean.

It must be clearly defined where the tattooing is done and where the waiting customers or accompanying friends should be seated.

\section{Getting Started with the Tattoo}

In the following, it is assumed that the professional tattoo artist follows the guidelines for good hygiene, which is described in one of the other chapters of this book.

The placing of the motive on the customer's body can be done in different ways. The most common ways are stencil or freehand drawing with skin markers - or a combination of both (fig. 2).

When the customer and the tattoo artist agree about the final design and placement of the tattoo, the actual work with the tattoo begins (fig. 3).

Nowadays, the tattoo artist can use different kinds of machines and needles, which is described in another chapter of this book.

It is important to use the right products in this process. Quality products. It does matter whether you work with a professional machine for USD 600 or a Chinese copy for USD 10. There is a difference. The expensive machine will transfer the ink with accuracy and uniformity into the customer's skin, whereas the Chinese copy typically works with irregularity and damages the skin.

The same applies for the needles. More expensive needles, purchased through a recognised dealer, work better and safer than the cheaper Chinese-copy products. You, as a tattoo artist, can directly import from the manufacturer.

You might be lucky to find one in a hundred cheap needles that work. However, the quality 
Fig. 2. Example of a combination of stencil and freehand drawing using a skin marker.


Fig. 3. Example of the process, from start to finish. First freehand drawing using a skin marker pen then outlining - black and shading - and finally colours: Voila! varies too much for you, as a tattoo artist, to consistently create quality work from time to time.

The same applies for the choice of colours. It's very important that you, as a professional tattoo artist, buy your colours at a dealer that you have knowledge about and trust. The market is filled with poor copies.

All of the major, recognised colour manufacturers find that their products are being illegally copied in countries such as China or Brazil. You can, as a user of the products, not tell the copies from the original. The only way you can be sure of what colours to use is to buy from a dealer approved by the manufacturer or to buy directly from the manufacturer.

The problems with the copy colours are, first of all, the manufacturer's product-sheets, which, of course, does not apply to the product you have purchased. So, you know nothing about what you are putting into your customer's skin. However, the analysis of the Chinese colours clearly shows that the manufacturer doesn't care at all. Several 
Fig. 4. Example of a poorly done tattoo - and the professional tattoo artist's fairly successful saving.
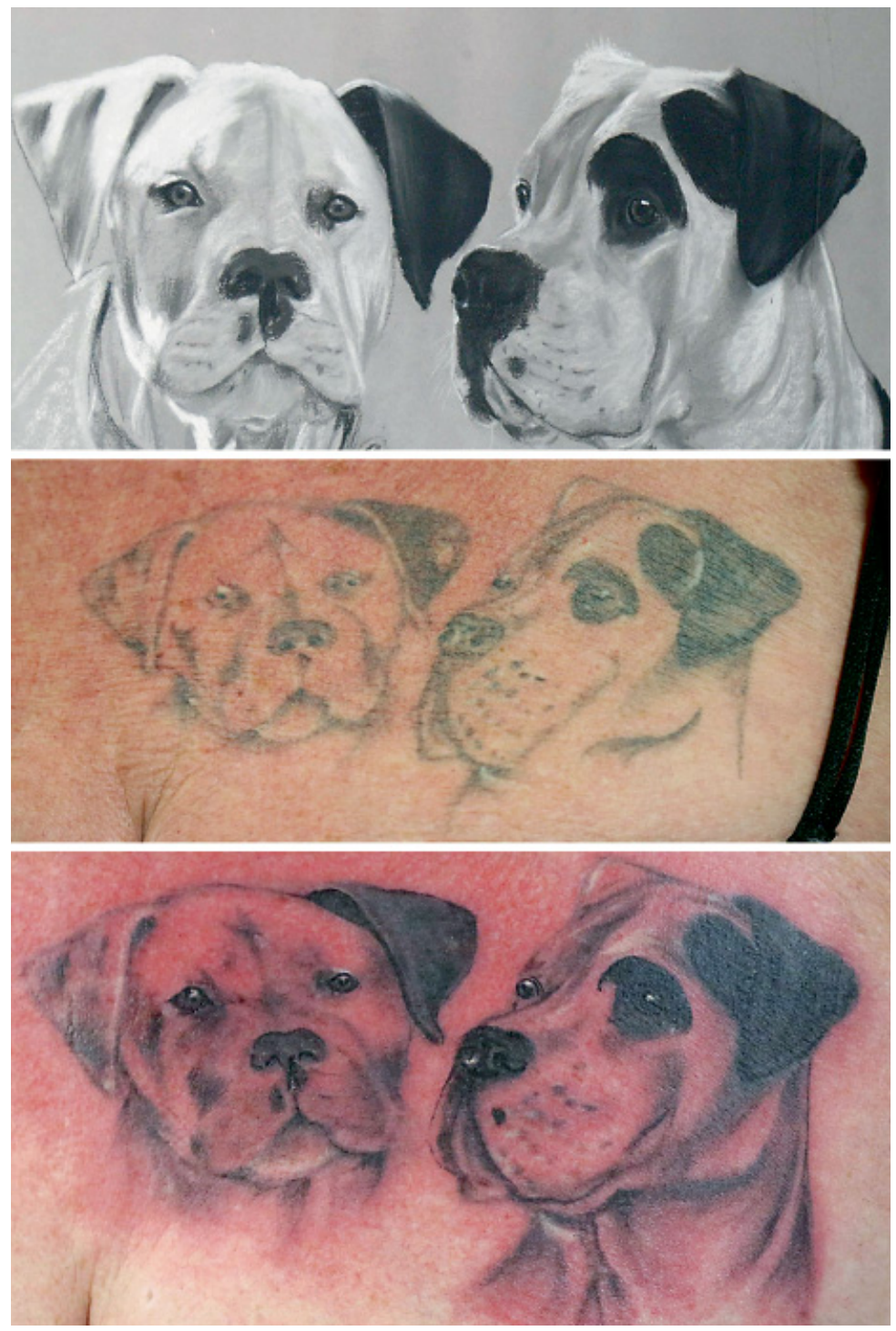

examples of contamination/bacteria in colours and on needles from dubious manufacturers have been found, and these products were sold as sterile.

\section{Working on the Tattoo}

While working on the tattoo, there are some basics you should be aware of. It's important to work at the correct depth of the skin. If you work too deep, you risk the colour flowing into the skin, giving a blurry, unclear outlining of the tattoo. However, if you don't work deep enough, the colour will disappear, and the customer will be left with a bad result, where parts of the tattoo will disappear as soon as the healing process is over. In the same way, it's important to work with a sufficient amount of colour in the needle tube so that the colour constantly flows evenly into the skin. 
Fig. 5. Portrait of a Papillon - done by a professional.
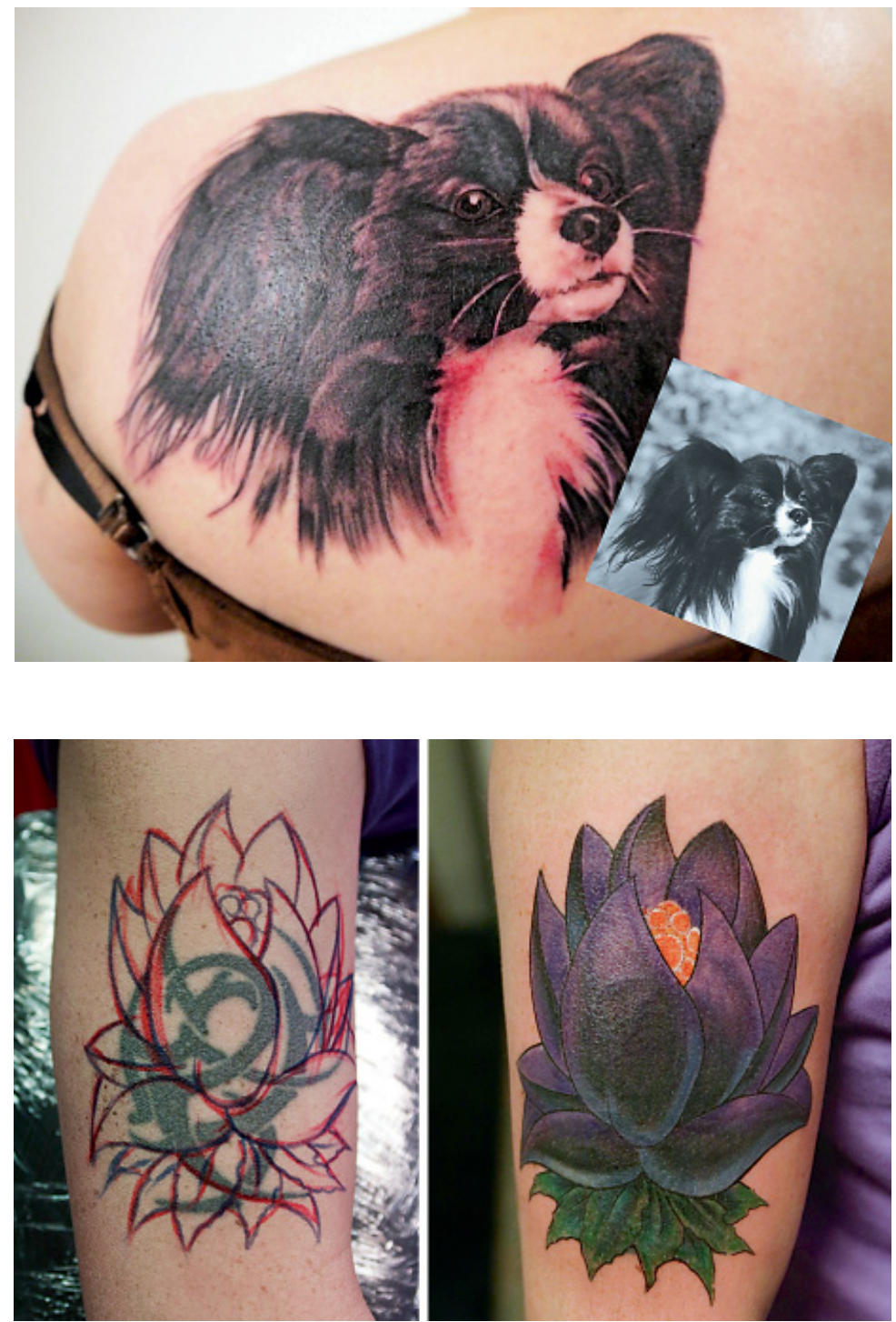

Fig. 6. Cover-up of an old black tribal-tattoo. Today, modern colours can, with some success, cover old black tattoos.
If you don't ensure this, you will to work too long on a single spot to fully colour the tattoo, with the skin being exposed to undue stress, and this will cause a prolonged healing process.

Especially, when you are doing a cover-up of an old tattoo, you must be aware of these things. First, the skin is already influenced by the old tattoo and therefore is more sensitive to the stress it's being exposed to by the new tattoo. In addition, you generally have to work harder and longer with the nee- dle to cover up the old tattoo, so the total impact on the skin is on average larger on cover-ups than on new tattoos made on untouched skin (fig. 4-6).

\section{Aftercare}

When the tattoo is done, the professional tattoo artist will make sure to advise the customer about taking care of the new tattoo. The new tattoo should 
Fig. 7. From something that looks like random lines on a leg to the final result: Art!
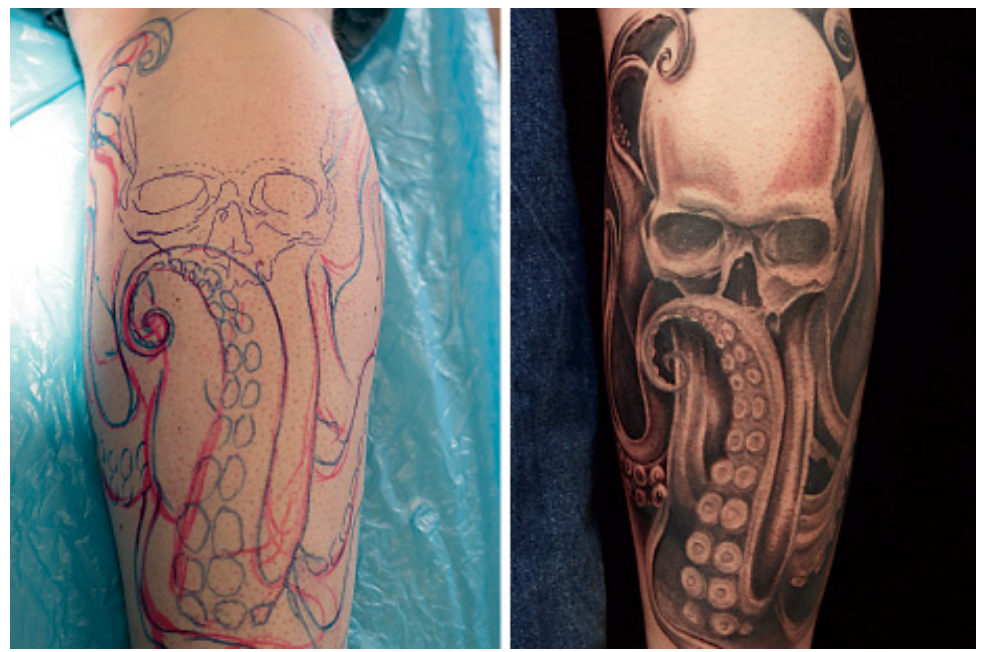

be kept clean with a sensitive, antiseptic and neutral soap. The tattoo needs to be lubricated 3-4 times daily with a product specifically for this purpose - typically an ointment containing panthenol. The customer should lubricate the tattoo until it has healed. In most cases, this will take about a week. Many studios hand out patient guidelines to the customer, where everything is described. Here, you can inform the customer on how to behave if any complications show up regarding the tattoo as well as give contact information for the studio.

\section{Art and Craftsmanship in Union}

Many of the professional tattoo artists you meet today make great art when they are working. However, the art is the finished tattoo (fig. 7), and the process behind it is good, old-fashioned craftsmanship.

The professional tattoo artist is a good craftsman who perfectly knows his equipment and materials and therefore becomes a person who makes art. 